\title{
Intra-cavity Frequency Doubling of an Electrically Pumped Edge-emitting 980 nm Laser Diode with PPLN
}

\author{
Kang Li ${ }^{1}$, Aiyun Yao ${ }^{1}$, N.J.Copner ${ }^{1}$, C.B.E.Gawith ${ }^{2}$ and Ian G Knight ${ }^{3}$ \\ 1. Faculty of Advanced Technology, University of Glamorgan, Pontypridd, CF37 1DL, UK \\ 2. Covesion Ltd., Romsey, SO51 9AQ, UK \\ 3. Bookham Technology Plc, Caswell, NN12 8EQ, UK
}

The growing trend for laser-based projection displays makes intra-cavity frequency doubling (ICFD) of electrically and optically surface emitting diode lasers in the near IR region become more interesting [1-3]. In this letter, we demonstrate for the first time to our knowledge ICFD of an edge emitting laser diode using a periodically poled lithium niobate (PPLN) bulk crystal, which has the potential to be scalable to high production volumes and low costs with immense implication for industrial applications.

Our new cavity design uses an external dichroic mirror coated for high reflectivity in the near-infrared range and transparent for blue light emission is used as output coupling mirror. Ultra-low reflectivity coating on the output facet of a Bookham single emitter laser diode gives less than $0.1 \%$ in the wavelength range of $976 \pm 15 \mathrm{~nm}$, which eliminates the original diode laser cavity allowing the extended longer laser cavity to dominate. The laser output is imaged through a single micro-lens such that the fast and slow axes beam waist give a near symmetrical $24 \mu \mathrm{m}$ beam radius focused to the midpoint of a $10 \mathrm{~mm}$-long $5.0 \mu \mathrm{m}$ period PPLN crystal supplied by Covesion. The beam waist is imaged through a $3 \mathrm{~mm}$ focal length lens such that retro-reflection of the IR light is achieved at the output coupler, providing superior stability. A thin film narrow bandwidth IR filter is inserted in the cavity before the PPLN to restrict the spectral laser bandwidth to $<0.1 \mathrm{~nm}$ so that optimal frequency conversion can be obtained. We have derived a model for the CW ICFD based on applicable laser rate equations which describe the second harmonic (SH) power $P_{2 \omega}$ for our system as:

$$
P_{2 \omega}=2 \cdot \frac{h v}{4 \beta^{2} \tau^{2}}\left[\frac{(K+\sigma \beta \tau)}{\sqrt{K}}-\sqrt{\frac{(K+\sigma \beta \tau)^{2}}{K}-4 \beta \tau\left(\sigma-\beta I_{e} \tau\right)}\right]^{2}
$$

where $h$ is Plank's constant, $v$ the fundamental frequency, $I_{e}$ is pump injection current divided by electronic charge, $\beta$ the stimulated emission rate, $\sigma$ includes losses and gain threshold, $\tau$ is the carrier lifetime, and $K$ is the nonlinear coupling coefficient.

Figure 1(a) shows that the experimental data is fitted to the numerical simulation when the cavity loss is up to $80 \%$. The high cavity loss is due to the non-ideal microlens used to create the symmetrical beam waist position in the PPLN. Even with this high intracavity loss we were able to demonstrate a highly stable CW output power of $0.02 \mathrm{~W}$ at $1.0 \mathrm{~A}$ injection current with $<5 \%$ variation in optical power over several hours. These powers were measured after an IR filter and with a PPLN temperature of $100^{\circ} \mathrm{C}$. Figure. 1 (b) shows the SH $(490.2 \mathrm{~nm})$ and IR spectra. We will discuss how more stable and efficient ICFD of an edge emitting laser could be achieved by enhancing the properties of anamorphic lens, focusing lens, IR filter and polarisation control. Higher brightness red-green-blue lasers can be expected using ICFD of CS-mount edge emitting laser bars composing of dozens of emitters with matched design components. These results demonstrate for the first time to our knowledge that stable ICFD is possible using telecom high power laser diodes with PPLN and that our model correlates with the experimental data. Further improvements are now possible through careful design of the lens used on the fast and slow axis beam waists and use of lower-temperature MgO-doped PPLN. This research could lead to disruptive lighting opportunities in laser-based projection displays.
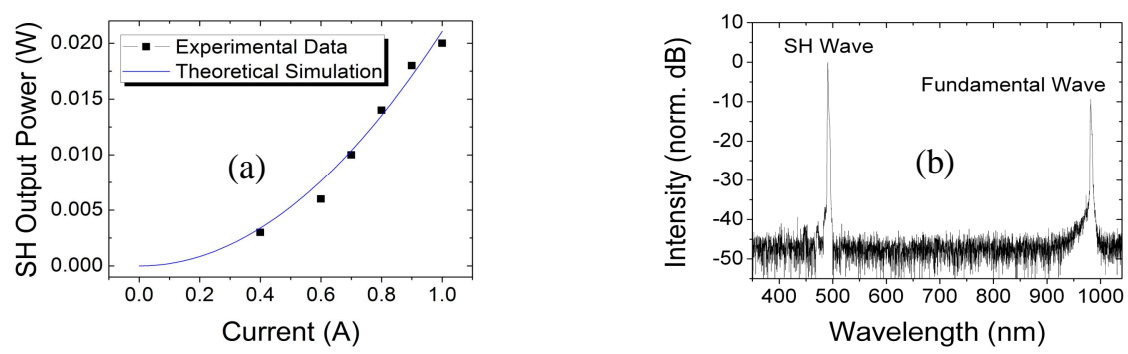

Fig. $1 \mathrm{SH}$ output characteristics as a function of the input current (a) and its output emission spectrum driven at 1.0 A (b).

\section{References}

[1] A. V. Shchegrov et al., "532-nm laser sources based on intracavity frequency doubling of extended-cavity surface emitting diode lasers", Proc. SPIE, 5332, 151 (2004).

[2] J. P. Watson et al., "Laser sources at $460 \mathrm{~nm}$ based on intracavity doubling of extended-cavity surface-emitting lasers," Proc. SPIE, 5364, 116 (2004).

[3] R. Hartke et al., "MgO-doped PPLN with cascaded structure for intracavity frequency doubling of optically pumped semiconductor disk lasers," CLEO/Europe and IQEC 2007 Conference Digest, paper CD8_2 (2007). 